Case Report

\title{
Maternal Fetal Trauma: When One Patient Becomes Two: A Case Study
}

\author{
Bonnie McCracken * \\ Visionem, Formerly of University of California Davis Medical Center, California, USA
}

\section{Article history:}

Received: September 5, 2019

Revised: December 23, 2019

Accepted: January 2, 2020
${ }^{*}$ Corresponding Author:

Bonnie McCracken

Visionem, Formerly of University of California

Davis Medical Center, California, USA

E-mail: bmccracken826@me.com

\begin{abstract}
Trauma is a complication in an estimated $6-7 \%$ of pregnancies, and is the most frequent cause of obstetric death. Trauma has been shown to result in maternal or fetal death, and in cases of extensive trauma, both may die. Management of pregnant patients has multiple challenges. It requires coordination of the trauma and obstetrics teams, as well as other specialties specific to the injuries of the patient. Management of the pregnant female has been described in trauma literature, but there are few reports on the management of both mother and fetus, when both have sustained trauma injuries. This case study discusses the series of events when a mother and fetus have injuries, and the considerations required in the post-resuscitative period when both patients survive.
\end{abstract}

Keywords: fetus, injury, pregnancy

\section{Introduction}

In trauma, rarely has a case been reported when serious injuries to the mother and fetus occur with positive outcomes. Pregnant females with trauma result in high maternal and fetal mortality, up to $73 \%$ following penetrating abdominal injuries and $66 \%$ in maternal blunt pelvic injuries [1]. While mortality can be high following trauma, nonfatal injuries also occur. Research indicates 1 in every 12 pregnancies survive nonlethal trauma with the most common mechanism being motor vehicle crashes [2,3].

Successful patient outcomes in trauma are dependent upon coordination of multiple teams, and preparation for complex high-risk circumstances. Advanced trauma Life Support (ATLS) algorithms may assist in guiding this coordination and resuscitation $[4,5]$.

Trauma to the pregnant patient presents challenges to trauma care, but is more complex when trauma affects both mother and neonate. The following case presented to University of California Davis Medical Center, a Level 1 adult and Level 1 pediatric hospital.

\section{Case Report}

A 23-year old woman (Ms. X) presented to a Level 1 trauma center after a highway motor vehicle crash (MVC). She was 33 weeks pregnant. The initial report indicated she was rearended at high speed and propelled into a concrete barrier. She was restrained by a 3 - point seatbelt and positive airbag deployment. Paramedics established intravenous therapy with a large bore IV administering isotonic crystalloid solutionat the scene. Her ankles and wrists were splinted due to the injuries sustained. She was normotensive, tachycardic and fetal heart tones (FHT) were present. Her chief concern was for her fetus. Upon arrival at the medical center, she was coded as a highlevel trauma patient, and was received in the resuscitation bay by the trauma team and Emergency Department (ED) team including an attending Trauma fellow, a 3rd year resident, an intern, a medical student, and ED nursing staff. Obstetrics (OB) team arrived shortly after.

\section{Resuscitation of the primary patient}

Ms. X. was awake, alert and verbal in the resuscitation bay. Vital sgns: normotensive (123/79 mmHg), tachycardic (116 
bpm), and slightly tachypneic (24 breaths per minute). Her chief complaint was chest, abdominal, and arm pain, and ongoing concern for her unborn child.

Her ED treatment course followed ATLS algorithms. Her examination was significant for abrasion on the lower chest wall, and an abrasive open wound on her left lower gravid abdomen.

She remained on isotonic crystalloid solution intravenously. Trauma lab panels were drawn. She had leukocytosis (17.3) commonly observed in trauma patients. Her initial hemoglobin and hematocrit were 11.9 and 34.2 respectively. All other laboratory tests were within normal parameters. The Kleinhauer Betke (KB) stain was positive for fetal blood in the mother's circulation, but this test result was not immediately available. KB stain is a test commonly performed after trauma measuring the amount of fetal hemoglobin transferred into the mother's bloodstream. It indicates placental hemorrhage, and can also be a predictor of preterm labor [6].

\section{Radiology}

- Chest X-ray: Left second and right 6-7 rib fractures.

- Chest Computerized Axial Tomography (CT): Sternal fracture, right pulmonary contusion, T3-4 compression fracture.

- CT Abdomen: Left lower flank laceration and soft tissue contusion, nondisplaced right 7 th anterior rib fracture, a mildly displaced fracture of the left transverse processes of L1 and L2, comminuted displaced impaction fracture of left

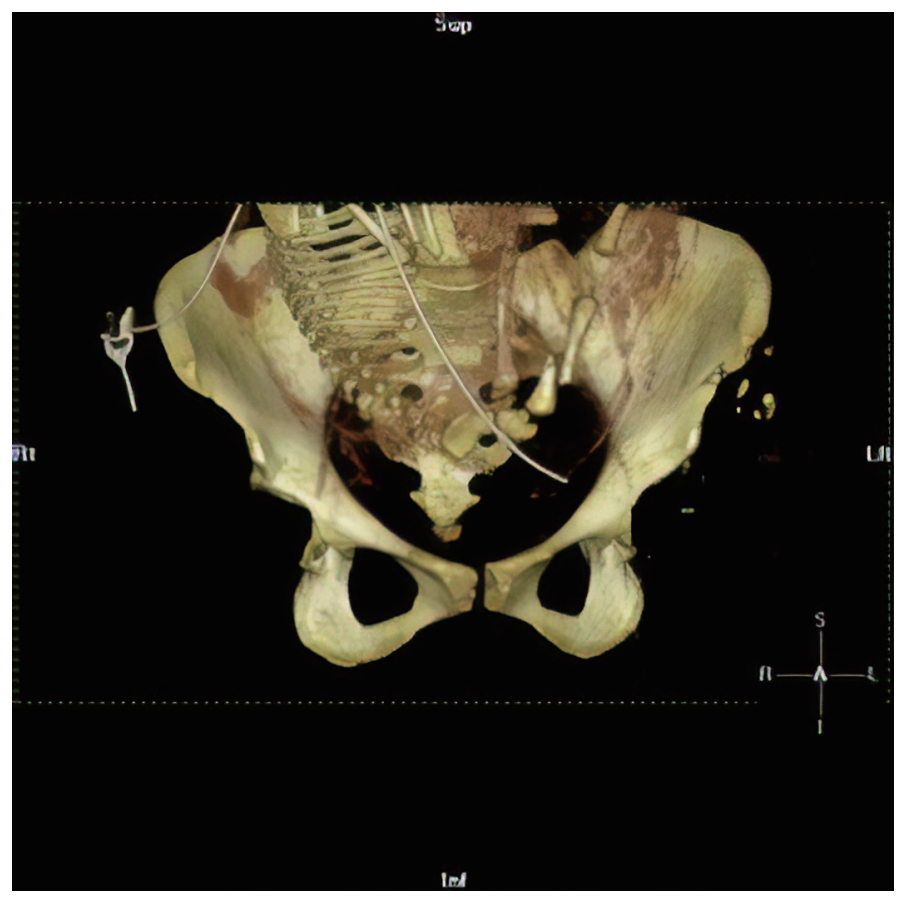

Figure 1. 3D reconstruction of the abdominal CT scan showing fetal femur fracture and maternal iliac wing fracture. $\mathrm{CT}=$ computerized axial tomography. iliac wing anteriorly, and a gravid uterus with single fetus in breech presentation. Fetus with a femur fracture (Figure 1).

- Extremity films: Right displaced comminuted DR fracture, right proximal phalanx fracture of the thumb, bilateral medial malleolar fractures.

- CT Head and cervical spine: Negative for traumatic injury.

During resuscitation, OB was attentive to the fetus. The FHT were 170 with minimal decelerations. Fetal ultrasound revealed a fetus in breech position 33 4/7 weeks gestational age, with what appeared to be a normal placenta. Presence of a femur fracture was duplicated on the CT scan. Fetal monitoring continued for ongoing evaluation, allowed for rapid intervention if neonatal distress was observed.

Orthopedics attended to the maternal fractures. Reduction of all fractures proceeded. The ankles were splinted, however the wrist required further reduction under sedation.

During the reduction, fetal distress was indicated by nonreassuring FHT with decelerations. With consultation and coordination from the attending trauma, OB and orthopedics teams, decisions were made to proceed to the operating room for a cesarean section, exploration of the abdominal wounds, and fixation of the multiple extremity fractures.

\section{Operative phase}

With the trauma surgeon leading the operation, Ms. X was taken to the operating room for an exploratory laparotomy and delivery of the fetus. Coordination and attendance of all the following teams were required in the OR: Trauma surgery, orthopedic surgery, $\mathrm{OB}$, anesthesiology, and Neonatal Intensive Care Unit (NICU) represented by a neonatal nurse practitioner and neonatologist. A midline incision was performed with a low transverse cesarean section for the delivery of baby $\mathrm{V}$. Placental abruption was noted, and the uterus was closed normally. Evaluation of the patient indicated that trauma continued. The devascularized tissue was debrided, and an evaluation for further intrabdominal injuries was negative. Orthopedics debrided the underlying iliac bone fracture, and the abdomen was closed with a Penrose drain in place. The orthopedic team continued with fixation of all her fractures. She was then transferred postoperatively to the surgical intensive care unit (SICU).

\section{Fetal resuscitation}

After delivery of the neonate, care was transferred to the NICU team. Baby boy V was flaccid and cyanotic with no respiratory effort and a heart rate of over 100 . He received 30 seconds of continuous positive airway pressure, followed by intubation due to insufficient intrinsic respiratory effort.

An emergency umbilical venous scores were 2, 5, and 7 respectively and he was transferred to the NICU. 


\section{Post resuscitative period- mother}

The mother's injuries :

- Concussion

- Bilateral Rib fractures

- Pulmonary Contusion

- Sternal fracture

- Blunt trauma to abdomen

- T3-T4 vertebral compression fractures

- Lumbar transverse process fracture of L1 and L2

- Closed fracture of (thoracic) vertebra endplate

- Bilateral Medial Malleolar fractures

- Right radial ulnar fracture

- Right Nondisplaced proximal phalanx fracture

- Placental rupture leading to need for immediate C-section

- Positive Kleinhauer betke

Consideration was given to medications prescribed to the mother as she was expressing breast milk to be given for her newborn son. Team coordination was required with the NICU team. While many of the medications she was given were "safe" whilst breast feeding, an extra level of vigilance was required because her baby was a premature trauma patient who was also receiving medication. Much of this coordination was through the nurse practitioners on the adult trauma team and the NICU team.

After a short course in the SICU, she was transferred to the Trauma Nursing Unit, a specialized unit for trauma patients. She received speech therapy evaluation for concussion, with no major cognitive deficits found. She had no respiratory sequela from her pulmonary contusion, rib, and sternal fractures. Pulmonary hygiene consisted of incentive spirometer use, ambulation, and coughing. Her degloving injury was closed with secondary intention as the Penrose drain was gradually advanced

Neurosurgery was consulted for her multiple vertebral fractures, no intervention was required. She only required pain management.

Orthopedics followed all fracture management. Open reduction internal fixation to her ankle fractures bilaterally and was nonweight bearing bilateral .She was non-weightbearing through her right wrist and hand. Physical and occupational therapy was needed for wheelchair transfer training, and basic ADLs.

Low molecular weight heparin, transitioning to warfarin was given to prevent clots Although very low levels of warfarin appear in breast milk, coordination with the NICU was required because the neonate receiving this breast milk was also a trauma patient.

Psychologically, this young mother needed access to her baby for maternal neonatal bonding and reassurance of the health status. She was taken to the NICU daily through the coordination of nurses and physical therapists. Holding her baby was difficult as she had upper extremity injures precluding her from holding the baby on her right side. The neonate also had injuries, which made it difficult to hold the baby to breast feed. This was eventually achieved with the help of the experienced neonatal nursing staff.

\section{Post Resuscitation- Neonate}

Baby Vs trauma diagnosis:

- Gestational age 33 4/7 weeks, premature infant (2.29 kilograms)

- Clavicle and femur fracture (Figure 2)

- Anemia due to acute blood loss

- Liver laceration

- Adrenal hemorrhage

- Kidney contusion

- Hypovolemia secondary to blood loss

Medical Diagnosis related to prematurity:

- Respiratory distress requiring mechanical ventilation

- Immature lungs requiring surfactant

- Persistent pulmonary hypertension

- Cardiac murmur with a small patent ductus arteriosus, left to right shunt

- Hypothermia requiring a heated isolette

Care required a multidipinary approach for this premature neonate trauma patient. The NICU staff, neonatologist and NICU nurse practitioners, directed the care with pediatric trauma consulting.

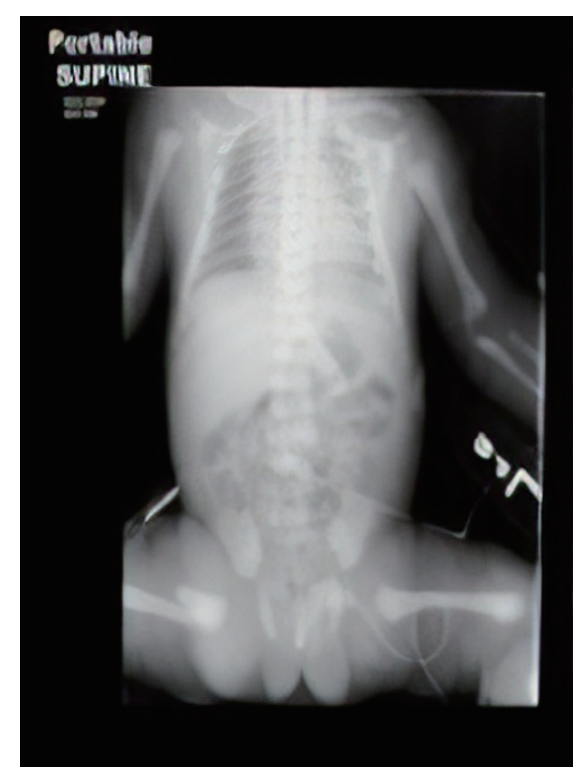

Figure 2. Neonatal femur fracture. 
Whilst there is a plethora of data regarding blunt liver injuries in adult patients, very little exists in the management of a neonatal patient [7]. Baby $\mathrm{V}$ had a $1.3 \mathrm{~cm}$ right lower posterior liver laceration and adrenal hemorrhage. Pediatric surgical management of these lacerations was observation by ultrasound. The hypovolemia caused by the bleeding led to hypotension with MAP 28-30. but in small amounts. The subsequent complication of fluid bolus was persistent pulmonary hypertension. Eventually it was determined that increasing circulating volumes perhaps was continuing to "pop the clot". In the end, lower volumes allowing permissive hypotension were key to the management of the neonatal intraabdominal hemorrhage.

A cranial ultrasound showed no intracranial or intraventricular hemorrhage. Due to the breech position of the infant, the acceleration deceleration force caused by the seat belt, lead to a femur fracture rather than a head injury.

The orthopedic trauma team was consulted on the neonate's femur fracture. Alignment was attempted with reduction of the femur unsuccessfully as it would not stay aligned. The neonate was double diapered to maintain a frog legged position, keeping the fragment ends in the same plane, allowing adequate healing of the bone. The mother had difficulty holding the neonate because of this position. Additionally, her inability to use her right upper extremity due to fractures, made skin to skin contact during holding was not possible.

Pain management in pediatric trauma patients can be difficult. In neonatal patients, pain is assessed using the Neonatal Infant Pain Scale this is based on facial expression, crybreathing patterns, arms, legs, and state of arousal. Considering this neonate was intubated and had multiple fractures, use of this scale was limited. Pain was managed by the finesse of the team and the nurses experience and expectation. Morphine infusions and intermittent injections of morphine were utilized for 15 days to manage the neonates pain. There also needed to be some consideration of the maternal intake of narcotics. Whilst small amount of morphine cross into the breast milk, and it is deemed safe for mothers to breast feed their babies, some consideration of transference to this premature injured neonate who was also being given narcotics was needed.

\section{Respiratory}

Baby V's pulmonary system did not transition normally due to the abrupt C-section, traumatic injury, and volume resuscitation. In a normal neonate at birth the pulmonary vascular resistance falls as the infant takes their first breath, and the pulmonary system fills with oxygen. By approximately 24 hours of life, the pulmonary blood flow increases to $100 \%$ of the cardiac output, and the pulmonary arterial pressure falls to approximately $50 \%$ of the systemic arterial pressure.
When this normal transition fails to occur as with baby $\mathrm{V}$, due to high volume resuscitation, an emergency C-Section, and failure of pulmonary artery pressure to decrease, the sequelae is persistent pulmonary hypertension.

Baby V was treated with surfactant to facilitate lung maturity and managed with multiple ventilator modalities. Facilitation of his ability to decrease pulmonary pressure over his NICU course was done with coordination of ventilation needs, fluid management and pain management.

\section{Cardiac}

The echocardiogram demonstrated normal intracardiac anatomy, with small patent ductus arteriosus, with a predominant left to right shunt. There was mild to moderate tricuspid regurgitation with a pressure gradient suggestive of systemic right ventricular pressure. There was a predominant, and persistent left to right shunt through PFO, with normal biventricular function. The intraventricular septum flattening, in conjunction with the echo cardiogenic findings, were consistent with persistent pulmonary hypertension. Although Sildenafil, a medication which relaxes muscles of the blood vessels, and increases blood flow to particular areas of the body was considered, the symptoms were resolved with an intervention of nitrous oxide. Normal cardia anatomy ultimately resumed normal function.

Baby V stayed for 23 days in the NICU without any adverse sequela. His mother was discharged from hospital after 10 days. Whilst she remained wheelchair dependent because she was non weightbearing bilaterally, and unable to use her right arm, she was present in the NICU every day after her discharge.

After discharge, both trauma patients had clinical follow-ups with multiple specialties. Baby V progressed well, and has met all developmental stages. His femur mended without any leg discrepancy. He had no neurological deficits and bonded well with his mother. Ms. X was able to weight bear bilaterally at 7 weeks post-operative and required outpatient physical therapy. Her abdominal injuries healed with secondary intention, without complication. She developed moderate to severe acute stress disorder. She was very fearful of driving or riding in a car and unable to return to work due to anxiety which manifested as panic attacks. She was referred for psychotherapy and started on medication. She continued to battle her anxiety for a year after her car crash.

Further information also was revealed regarding her crash. Initially this was reported as a highway rear-end accident. Upon presentation to the ER, the trauma team felt her injuries were more consistent with a frontal impact due to the acceleration/deceleration type injuries. It was discovered that another car crash occurred minutes before her collision. A driver charged with driving under the influence had entered the freeway driving in the opposite direction crashing into an 
oncoming car. This car was upending into its roof and landed in the middle of a traffic lane. This car was not seen by Ms. X a bend in the road. She struck this car head-on and was then spun into the concrete barrier. The fact she wore her seat belt, and her airbags were deployed, prevented fatal injuries from occurring.

Additionally, baby $\mathrm{V}$ was in a breech position which was revealed to Ms. X at an obstetric check the week before the car crash. At this point in her pregnancy, the fetus would normally be turning into a head down position. At her previous obstetric appointment, she was shown a technique to help the fetus turn to a head down position, called external cephalic version. She admitted to not following these instructions because it was uncomfortable. If this fetus was in a head down position, the outcome may have been worse. The amount of energy that fractured his femur and caused abdominal injuries would have been transferred to his cranium. In the end, the outcomes of both mother and neonate were excellent. Both recovered with no physical sequela.

\section{Discussion}

The outcome of trauma in pregnancy is not always this good. In the United States, trauma is the most frequent cause of nonobsterical maternal death [1-3]. Resuscitative goals in the pregnant trauma patient are the same for any trauma patient. However, the resuscitation may be more complex due to the normal changes of the female during pregnancy. The normal physiological changes that occur in pregnancy lead to more difficult detection of a patient in distress. The normal increase in maternal cardiac output, and blood volume in the pregnant patient, mask the classic signs of hypovolemia until up to $40 \%$ of blood volume has been lost [1]. In a pregnant patient after the first 20 weeks, there is also the consideration of the viable fetus, who may or may not be showing signs of stress or injuries. The ongoing mantra in the pregnant patient is always treat the mother first [2].

In trauma, a heightened concern of radiation exposure exists, especially in early trimesters with the possible impact upon the development of organs. This needs to be assessed with the need for accurate evaluation of the traumatic injuries. A CT scan of the abdomen may be required when there is concerns for identification of maternal abdominal injuries $[2,6]$. The risk benefit must be considered at all times. While there is less danger of complication to radiation exposure in the last trimester of pregnancy, careful consideration is required [2].

Excellent outcomes in trauma patients are dependent upon the skills of not only the trauma team, but in all the consultants, teamwork, and anticipation of the patient needs. Coordination of care among all team members is imperative to a successful outcome [2-4]. In this case, coordination of care continues to be an important aspect of the post resuscitative phase of care as 1 trauma patient became 2 trauma patients.

\section{Conflicts of Interest}

The author has no conflicts of interest to declare.

\section{References}

[1] Petrone P, Talving P, Browder T, Teixeira PG, Fisher O, Lozornio A, et al. Abdominal injuries in pregnancy: A 155-month study at two level 1 trauma centers. Injury 2011;42(1):47-9.

[2] Jain V, Chari R, Maslovita S, Farine DJ. Guidelines for the Management of a Pregnant Trauma Patient. Obstet Gynaecol Can 2015;37(6):553-74.

[3] Barraco RD, Chiu WC, Clancy TV, Como JJ, Ebert JB, Hess LW, et al. Pregnancy and Trauma. Eastern Association for the Surgery of Trauma. J Trauma 2010;69(1):211-4.

[4] Henry S, Brasel K, Stewart, RM. ATLS® Advanced Trauma Life Support; Student Course Manual, 10th ed. Chicago (IL); ACS American College of Surgeons: 2018.

[5] Agency for Healthcare Research and Quality (AHRQ 2012) [Internet]. The Quick Reference Guide to TeamSTEPPS $®$ Action Planning: TeamSTEPPS $®$ Implementation Guide. Rockville (MD); AHRQ: 2008. Available from: https://www.ahrq.gov/sites/default/files/publications/files/lepguide.pdf.

[6] Muench MV, Baschat AA, Reddy UM, Mighty HE, Weiner CP, Scalea TM, et al. Kleinhauer-betke testing is important in all cases of maternal trauma. J Trauma 2004;57(5):1094-8.

[7] Coimbra R, Hoyt DB, Engelhart S, Fortlage D. Non operative management reduces the overall mortality of grades 3 and 4 blunt liver injuries. Int Surg 2006;91(5):251-7. 